\title{
The Motivated Brain: Insights from Neuroimaging Studies of Human Male Sexual Affiliation Context
}

\author{
Harold Mouras*,1 \\ ${ }^{1}$ Laboratoire de Neurosciences Fonctionnelles et Pathologies, UFR de Médecine, Université de Picardie Jules Verne, 3 \\ rue des Louvels, 80036 Amiens Cedex 1, France
}

\begin{abstract}
The advent of functional neuroimaging techniques has allowed to address the question of the role of the brain in a new light, being now able to record brain activity under different kinds of perceptual, cognitive or motor paradigms. Two exponentially emerging fields, i.e. social and affective neurosciences, converge on topics such as brain processing of emotional information issued by the congeners. As any social interaction obbeys a motivational dimension of interattraction, it is therefore important to study the role of the brain in specific functional contexts. In this paper we show how the emergence of a new field crystallized around the study of brain circuits involved in sexual affiliation has helped providing important results to understand the brain's role in social motivated interactions. Specifically, these studies show for this involvement a central physiological component and its cortical representation that seems to be essential for social interactions with motivational component.
\end{abstract}

Keywords: Motivated brain, neuroimaging studies, social interactions.

\section{INTRODUCTION}

Along the last two decades, the constant development of neuroimaging techniques allowed to observe alive brain activations in different kind of perceptual, cognitive and motor paradigms. Two particularly emerging fields are affective neurosciences [1], interested in understanding the neural processes involved in emotional and motivational processes and social neurosciences [2] focusing on the cerebral networks involved in social stimuli cerebral processing. These two exciting fields are converging on the question of the cerebral processing of emotional informations associated to stimuli issued by congeners. In this domain, recent studies have shed light on a certain correspondance between cognitive and neural systems involved in action perception and production. For instance, a subgroup of neurons of the premotor cortex, mirror neurons, were reported as increasing their firing rate during action production on one hand and observation of the same actions realized by a congener on the other hand [3]. At the moment, this topic is extending to the emotional domain, leading to studies on the neural correlates of empathy and associated perception-action coupling mechanisms [4]. Feeling an emotion when observing it in a congener involves a physiological synchrony between the observer and the observed people [5]. For example, exposition to a disgusting flavor activates the same brain structures (notably the insula) than the observation of a people presenting a facial expression of disgust [6]. Insular lesion prevents both disgust experience and ability to recognize social signal conveying disgust [7]. As well, neural circuits of nociception are

*Address correspondence to this author at the Laboratoire de Neurosciences Fonctionnelles et Pathologies, UFR de Médecine, Université de Picardie Jules Verne, 3 rue des Louvels, 80036 Amiens Cedex 1, France;

Tel: +33 3228254 42; E-mail: hmouras@gmail.com activated either by pain itself or by the presentation of the affective state of a subject in a painful situation $[8,9]$. These results suggest that neural circuits involved in emotional information processing participates in intersubjectivity engaged in interacting people. However, the spatial recovery is not perfect leading to a dissociation between regions involved in self or other perception. Such dissociation participates to avoid a confusion between self and other and then, the expression of an excessive empathy, that would be potentially problematic [10]. Interestingly, some brain regions play a key role in distinguishing between self-related versus other-related representations. Although empathy has an intrinsic social component, relationships between people first follows an interattraction motivational component (that can be either positive or negative). The notion of interattraction can be studied through different functional contexts; sexual motivation is one of these contexts. During the last decade, the study of neural networks involved in sexual information processing has been widely developped bringing results that can be partly generalyzed to social neuroscience. Only few studies were available till the end of the nineties. However, the field is now in exponential emergence. Through recent results, we would like to underline how this functional context has allowed to better understand the role of the brain in motivated social interactions. Studies mentionned here had clearly a double objective: (i) a better identification of the neural networks involved in visual sexual information processing; (ii) the use of the specificities of this response as a working model to study neural networks activated in social exchange situations. In order to articulate psychological processes and the corresponding neural correlates, this can be useful to propose a subdivision of visual sexual information processing into non-exclusive components that need to interact and co-occur to produce the resulting behavior. 
Therefore, these components can be gathered into a neurobehavioral model including: (i) a cognitive component, associated to identification and categorization of stimuli; (ii) an emotional component, related to the pleasure sensation which is linked to the pleasure associated to sexual arousal increase; (iii) a motivational component, linked to the processes involved in directing behavior towards a sexually relevant target; (iv) a physiological component, related to all physiological variations (i.e. genital, respiratory and cardiovascular responses). Therefore, this is important to bring together a psychological process and a cerebral activation and to explain how these processes articulate with the other components of the model.

Functional magnetic resonance imaging (fMRI) is characterized by a good spatial resolution but not so good temporal resolution. At the moment, this is the most widely used techniques for neuroimaging in cognitive neuroscience. For the functional context of sexual motivation, the use of this technique allowed a better identification of early cerebral processes involved in visual sexual information processing, being thought as included in the cognitive component of the model. Correlational analyses were also performed to study the covariation of cerebral hemodynamic signal and a very specific peripheral physiologic response, male genital's response that was only recently measured in the constrained experimental environement of fMRI. In this paper, two important aspects that allowed to identify psychological and neural processes will be presented. Interestingly, the obtained results had a broader extent that only related to the functional context of sexual motivation.

The speed and the nature of socioaffective information cerebral processings is an important question. Is this kind of information processed differentially than non-emotional information? For this purpose, a study [11] was conducted and differed from other studies by much shorter stimulation period and the use of photographic stimuli. This paradigm was constructed to induce a low-level sexual arousal, more adapted to identify early activated brain areas. Simultaneously to fMRI data acquisition, subjective reports along different dimensions (beauty, desire, pleasure, displeasure, interest, tenderness, intensity of perceived erection) were recorded. In this study, the presentation of decontextualized sexual stimuli with a scanner was validated. Participants reported significantly higher ratings in response to sexual visual stimuli than for neutral stimuli for the dimensions "beauty of the depicted character", "perceived desire to engage a relation", "perceived intensity of erectile response", "intensity of pleasure", "interest for pictures". Although the experimental setting and procedures were not ecological to study sexual arousal, behavioral results indicate a possible induction of sexual attitudes. Therefore, sexual affiliation could be used in future experiments as a working functional context to explore the neural correlates of motivated social interactions.

How about brain activations when looking at visual sexual stimuli ? The pattern of brain activations obtained in a group of eight participants is depicted on Fig. (1).

In accordance with our hypotheses, reported activations were linked to cognitive processes. They were identified in inferior parietal lobules (IPL, Fig. (1), c). These areas are known to be activated in monkeys during visual fixation episods directed to motivationally relevant targets as compared to visual fixation episods occuring during environemental exploration [12] or for example in cocaine users exposed to scenes where cocaine was used [13]. This early activation has been thought to be involved in (i) matching processes between visual information and body physiological state when receiving those visual informations and (ii) enhancing attentional mechanisms towards motivationally relevant targets. With the introduction of other temporal functions, activations have been reported in superior parietal lobules (Fig. 1, b and b'). These activations were nonetheless sustained during all visual sexual stimuli presentation period but also early as for activations recorded in IPLs. These results were in accordance with a very short categorization time for sexual stimuli [14] and early parietal activations in response to loved ones [15]. Therefore, these activations were interpreted as the anatomical support of an early amplification of attentional processes directed towards visual sexual stimuli.

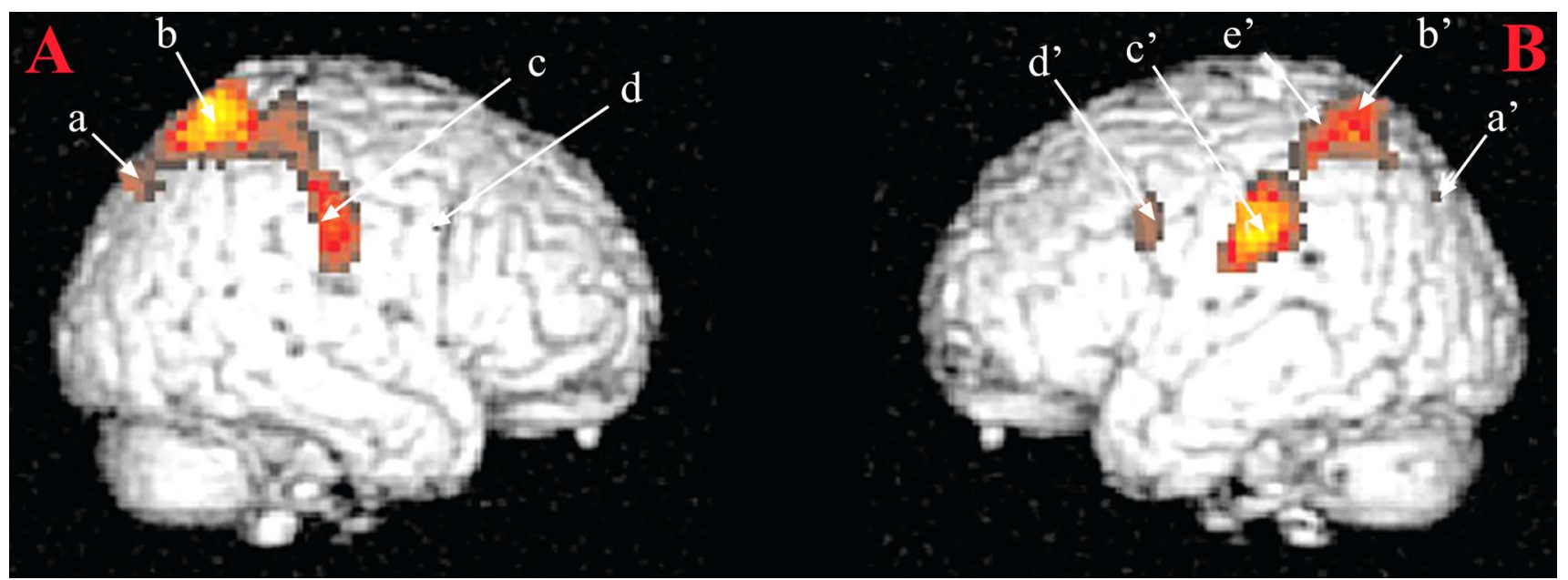

Fig. (1). Brain areas activated in response visual sexual stimuli as compared to neutral stimuli. Notes: (A) Right hemisphere: a: parietooccipital sulcus; b: superior parietal lobule; c: postcentral gyrus; d: precentral gyrus. (B) Left hemisphere: a: superior occipital gyrus; b: superior parietal lobule; c: inferior parietal lobule; d: precentral gyrus; e: intraparietal sulcus. These activations were obtained for a statistical threshold of $\mathrm{p}<0.05$, corrected for multiple comparisons. Figure from [11]. Reproduced with permission. 
Were the brain areas identified in this approach specific of sexual affiliation context ? Regarding this aspect, it was important to construct a specific MR-compatible device to be able to record volumetric penile plethysmography concurently to cerebral BOLD responses. This approach allowed performing correlational analyses between the genital and cerebral response's magnitude [16, 17]. Therefore, brain data were analyzed not only with models including hypothetical temporal functions to explain brain signal variations, but also with models that included functions indexing an objective physiological signal recorded simultaneously to brain activations. This allowed to take into account the individual aspect of each physiological response for brain data analyses, which is not often used in a classical neuroimaging statistical approach. Moreover, it should be noted that volumetric penile plethysmography is more reliable than circumferential penile plethysmography and seems to be more sensitive to low-level of penile response. This allowed a better detection of correlations between cerebral and physiological responses $[18,19]$. Therefore two studies were conducted, recently and for the first time, following such simultaneous approach [16, 17] with the aims to characterize the neural correlates of penile response initiation and to analyze precisely the temporal correlations between cerebral and genital responses in order to test several hypotheses on the relative temporal courses (distinction between areas involved in the genital response command and ones involved in its cortical representation) and to identify regions having a broader role than only involved in the physiological component of the model. A previous study reported a delay between the begining of visual sexual stimuli presentation period and the occurence of the physiological period [20]. Based on this observation, the hypothesis was made that brain areas leading the genital response would show an activation before the genital response itself and those involved in the cortical representation of the genital response would be activated after the genital response itself. Therefore, in a block-type paradigm including pictures related to different experimental conditions, cerebral and genital responses have been simultaneously recorded with the scanner. An interesting point is that one third of the participants with a recorded plethysmographic penile response did not report a corresponding subjective perception for low-level of induced sexual arousal. This is in accordance with a previous reported low correlation between subjective perception and real physiological response for such levels of arousal [19]. Three different temporal correlational analyses were performed between the BOLD signal and the volumetric plethysmographic penile response that were recorded either simultaneously, 20 seconds after in order to identify brain areas involved in genital response control and 10 seconds before in order to identify brain areas involved in genital response cerebral representation (Fig. 2).

Correlational analyses shed light both on very specific aspects of motivational information cerebral processing (regarding mainly the physiological component of the neurobehavioral model) and broader aspects of socioaffective relevant informations. Both aspects are underlined in the two following sections. First, on the physiological component of the model. In the medial prefrontal cortex (corresponding to Brodmann areas 9 and 10), several foci of activation were reported (Fig. 2, c and g), in agreement with anatomical studies showing projections from this region to diencephalic regions that controls erection, as the brainstem and spinal cord [21, 22]. Within this region, a regional disparity has been demonstrated with close activated foci, with some correlated positively, others negatively with the penile response, in agreement with similar observations for the galvanic skin conductance [23]. This result argues for a co-location of structures involved in the control and representation of physiological responses [24]. In another area, the insula, the correlation analysis showed a difference between the role of the anterior insula involved in controlling the physiological response (its stimulation induces changes in heart rate and blood pressure; [25]) and its posterior portion which is likely involved in the representation of the physiological response (this region is activated by manual stimulation of the penis [26]). Finally, within the anterior cingulate cortex, (Fig. 2 b), its caudal (posterior) seems to be involved in the control of genital response, whereas its rostral (anterior) portion seems rather involved in the inhibition of the erectile response (negative correlation between the signal BOLD and the erectile response). The correlational approach was also used to characterize the cortical representation of the penile response. Within the original homunculus [27] it stood at the paracentral lobule (Fig. 2 a). However, for two small clusters in the inner surface of the somatosensory cortex, the BOLD
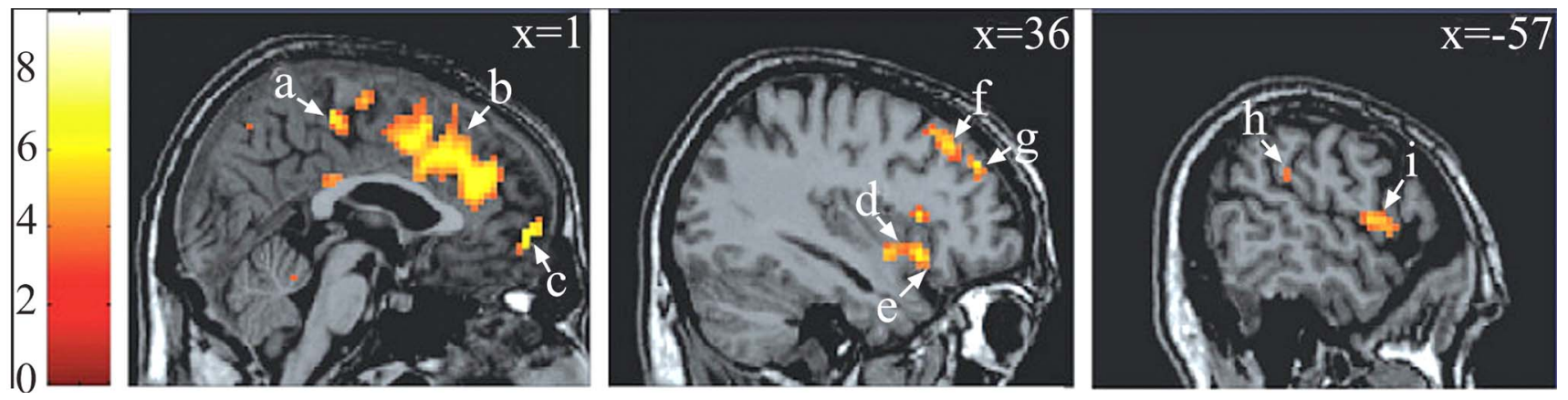

Fig. (2). Brain areas in which the BOLD signal was positively correlated with the plethysmographic signal with no lag. Notes : a $=$ paracentral lobule* (BA $5 ; 0-30$ 54) ; b = anterior cingulate gyrus* (BA $32 ; 330$ 33); $\mathbf{c}=$ medial fronatl gyrus* (BA $10 ; 1563-3$ ) ; $\mathbf{d}=$ insula (BA $13 ; 393$-9) ; e = inferior frontal gyrus* (BA $47 ; 3618-12$ ); $\mathbf{f}=$ middle frontal gyrus* (BA $8 ; 303348) ; \mathbf{g}=$ middle frontal gyrus* (BA $9 ; 364533$ ) ; $\mathbf{h}=$ inferior parietal lobule (BA $40 ;-54-3327$ ) ; $\mathbf{i}=$ precentral gyrus (BA $6 ;-5736$ ). Data were initially thresholded at $\mathrm{P}<0.001$ uncorrected. $* \mathrm{P}<=.05$, corrected for multiple comparisons. Reproduced from [17] with permission. 


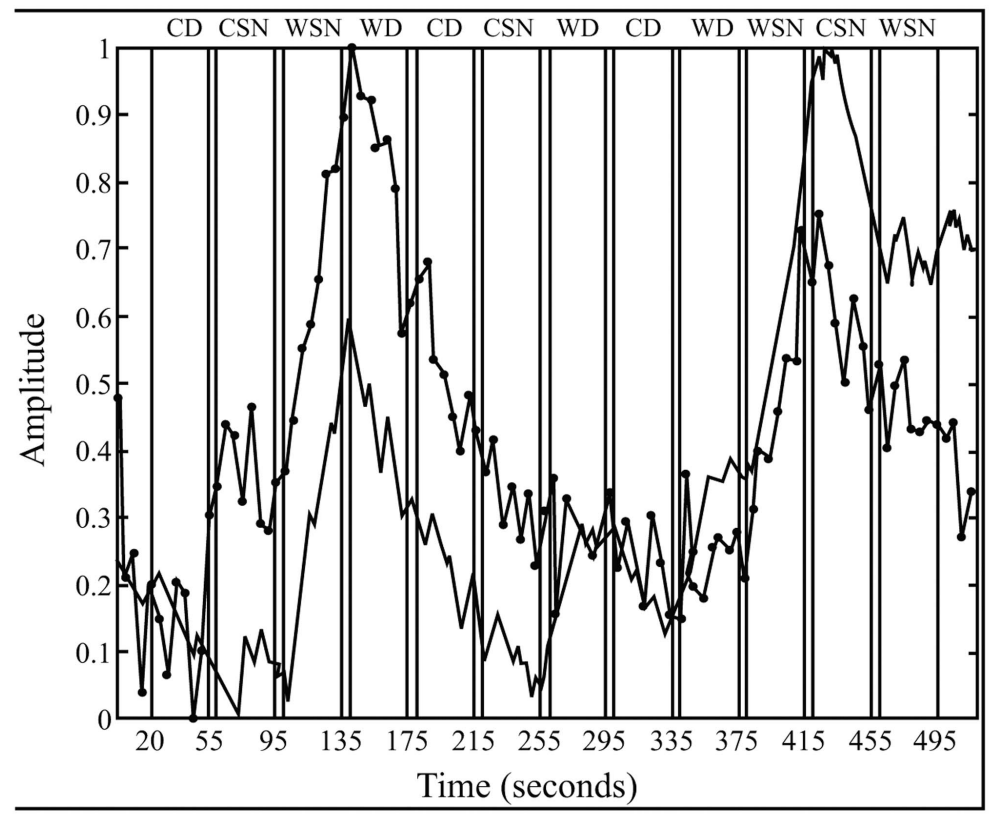

Fig. (3). Temporal courses of respectively the bold (black line) and penile plethysmographic responses within the right medial frontal gyrus (BA 10; $x, y, z=16,63,-3$ ) within the different experimental conditions. Blocks of "sexually explicit" pictures are indicated by the acronym WSN. Reproduced from [17] with permission.

signal co-varies with the plethysmographic signal and the surface extends substantially in the condition analysis which precedes the penile response of the BOLD signal 10 seconds. This is consistent with earlier imaging $[20,28]$ or cortical potentials in response to stimulation of the dorsal nerve of penis [29] showing cortical representation of the genitals more dorsal and lateral than conventionally reported [27]. Thus, the results of this first study argues for a double cortical representation of genital response, one (lateral) can be linked to superficial sensory stimuli and the other (medial) rather related to the perception of penile tumescence itself. Finally, the correlational approach has highlighted the involvement of a network of frontal areas, parietal insular and cingulate cortex in the initiation and maintenance of the physiological response. In addition, manipulation of the time window between BOLD signals and plethysmographic suggests that the existence of subregions are sometimes very close with contrasting roles, sometimes conflicting. An example of respective temporal courses of the bold signal in a cerebral area and the one of the penile response as measured by volumetric penile plethysmography are depicted on (Fig. 3).

A recent study [30] demonstrated the involvement of the mirror neuron system in empathy, whereas these neurons are classically known to respond to the observation of movements performed by others. Consequently, it was important here to assess the degree of implication of these mirror neurons in the observation of sexual exchange. Therefore, our hypotheses were that in response to the presentation of sexual visual stimuli, the magnitude of activation of the brain networks involved in movement observation and motor imagery would predict the intensity of genital response; the level of the erectile response would predict the amplitude of the BOLD response in primary and secondary somatosensory areas corresponding to the projection of the penis.
Three results are developed here (see Fig. 4) illustrating how their generalization was possible for neuroscience of social interactions.

The first region in which the variations in BOLD signal were correlated to those of genital response was the left frontal operculum. This region is part of the mirror neuron system that encodes the correspondence between observed events and internally generated actions promoting a link between observer and the actor [3]. In the functional context of sexual affiliation two interpretations are possible regarding the role of this neural system: preparing to perform an activity similar to that observed and/or support of an internal representation of movement [31].

On the one hand, these neurons may play an important role in preparing the necessary action (in these experiments, participants often report episodes of motor imagery usually corresponding to imagined sexual acts). On the other hand, the behavioral response was also recorded by physiological (the erectile response) and some of the efferences of the frontal operculum to the insula could be the anatomical support. Therefore, these results place the physiological response at the center of the processes involved in social relations with a strong motivational component. In addition to mirror neurons areas, the correlational analyses identified several regions in the network of motor imagery and action observation $[32,33]$ including the inferior parietal lobule $[31,34]$ and the left supramarginal gyrus [33-36].

For somatosensory areas, important results have been obtained. For some areas (lower part of Brodmann 2), activation preceded the genital response, suggesting that these areas "flew" the physiological response, thereby pointing to the concept of somatosensory imagery [37]. Everything happens as if the observation of sexual explicit scenes just did not induce activation in motor areas corresponding to the hand, but also in areas of the 


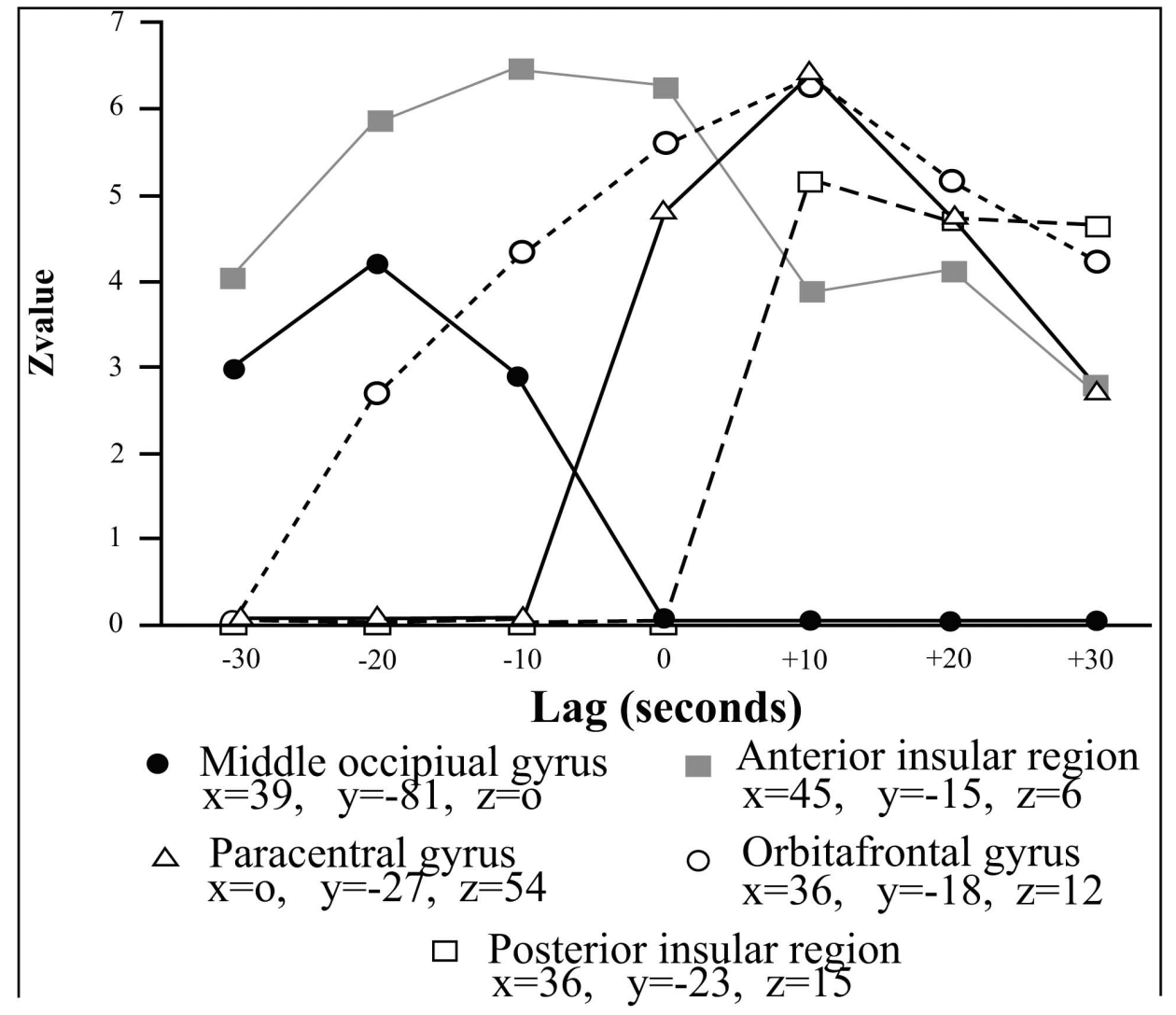

Fig. (4). Correlations profiles between the BOLD and the penile plethysmographic signal as a function of the temporal lag introduced in the correlational analyses between the two signals. The vertical axis corresponds to the $\mathrm{Z}$ value associated to the correlaton coefficient between the two signals and the horizontal axis corresponds to the value of the temporal lag. Positive lags correspond to those where penile plethysmographic response preceeded the BOLD signal; negative ones where the BOLD signal itself preceeded the penile response. Reproduced from [17] with permission.

somatosensory cortex corresponding to the representation of the hand. Such processes may play a role in recognizing emotions in others by the observer [38] or in preserving the sense of self during action observation [39,40]. Finally, for other regions, such as primary and secondary somatosensory areas, some of them seemed to "drive" the physiological response while others are "driven" by it, suggesting the existence of positive feedback loops during the establishment of the genital response.

As a conclusion of this paper, during the last years, it has been important to see the exponential emergence of a new field: the study of cerebral networks involved in motivated social interactions. This study has been allowed thanks to the use of very specific functional contexts such as sexual motivation. Importantly, specific studies first demonstrated the feasibility of using modern neuroimaging techniques such as fMRI to study brain networks involved in a decontextualized experimental setting. This feasibility has been demonstrated behaviorally and then, on the neural side. The major role of early attentional enhancement has been demonstrated and shed light on early cognitive processes involved in the cerebral processing of informations with a motivational dimension. However, the interest of the human male sexual arousal working model is to produce a very specific physiological response (i.e. penile response) that is important to record simultaneously to cerebral activations in order to perform highly precise correlational analyses that have been showed in several studies to produce very different results than more classical subtractive blockanalyses. On one hand several brain regions (medial prefrontal cortex, anterior insula, anterior cingulate cortex) were identified as playing a key role in the genital response command. On the other hand, the classical somatosensory cerebral representation of genital response was somehow completed. Until now, an unique representation was proposed in the original homonculus [27] but the studies summarized in this paper allowed to propose a second more lateral localization of this representation that could specifically involved in superficial sensory stimulations. These studies allowed to demonstrate the involvement of the mirror neuron system in sexual information cerebral processing and that the intensity of activation of this region and others involved in motor imagery predicts the genital response magnitude.

Finally, these results appear to be broader to identify the role of the brain in social interactions with a high motivational component putting at the center of this question its physiological component and its associated cerebral representation.

\section{ACKNOWLEDGEMENTS}

The author wants to acknoledge Maria Flavia Morel Bellini de Souza for the correction of this manuscript. This work was supported by grants from the French Ministry of Health 
and from the French Ministry of research (ACI Cognitique 2001). We thank J.P. Souteyrand for his technical assistance.

\section{REFERENCES}

[1] Pankseep J. At the interface of the affective, behavioral, and cognitive neurosciences: decoding the emotional feelings of the brain. Brain Cogn 2003; 52: 4-14.

[2] Insel TR, Fernald RD. How the brain processes social information: searching for the social brain. Annu Rev Neurosci 2004; 27: 697722.

[3] Rizzolatti G, Fadiga L, Gallese V, Fogassi L. Premotor cortex and the recognition of motor actions. Brain Res Cogn Brain Res 1996; 3(2): 131-41.

[4] Preston SD, de Waal FBM. Empathy: Its ultimate and proximate bases. Behav Brain Sci 2002; 25(1): 1-20.

[5] Levenson RW, Ruef AM. Empathy: a physiological substrate. J Pers Soc Psychol 1992; 63(2): 234-6.

[6] Phillips ML, Young AW, Scott SK, et al. Neural responses to facial and vocal expressions of fear and disgust. Proc Biol Sci 1998: 265(1408): 1809-17.

[7] Calder AJ, Keane J, Manes F, Antoun N, Young AW. Impaired recognition and experience of disgust following brain injury. Nat Neurosci 2000; 3(11): 1077-8.

[8] Singer T, Seymour B, O’Doherty J, Kaube H, Dolan RJ, Frith CD. Empathy for pain involves the affective but not sensory components of pain. Science 2004; 303(5661): 1157-62.

[9] Morrison I, Lloyd D, di Pellegrino G, Roberts N. Vicarious responses to pain in anterior cingulate cortex: is empathy a multisensory issue? Cogn Affect Behav Neurosci 2004; 4(2): 2708 .

[10] Batson CD, Sager K, Garste E, Kang M, Rubchinsky M, Dawson $\mathrm{K}$. Is empathy-induced helping due to self-other merging ? J Pers Soc Psychol 1997; 73: 495-509.

[11] Mouras H, Stoléru S, Bittoun J, et al. Brain processing of visual sexual stimuli in healthy men: a functional magnetic resonance imaging study. Neuroimage 2003; 20(2): 855-69.

[12] Lynch JC, Mountcastle VB, Talbot WH, Yin TC. Parietal lobe mechanisms for directed visual attention. J Neurophysiol 1977; 40(2): 362-89.

[13] Garavan H, Pankiewicz J, Bloom A, et al. Cue-induced cocaine craving: neuroanatomical specificity for drug users and drug stimuli. Am J Psychiatry 2000; 157(11): 1789-98.

[14] Spiering M, Everaerd W, Elzinga B. Conscious processing of sexual information: interference caused by sexual primes. Arch Sex Behav 2002; 31(2): 159-64.

[15] Pizzagalli DA, Lehmann D, Hendrick AM, et al. Affective judgments of faces modulate early activity (approximately $160 \mathrm{~ms}$ ) within the fusiform gyri. Neuroimage 2002; 16(3 Pt 1): 663-77.

[16] Mouras H, Stoléru S, Moulier V, Pélégrini-Issac M, Rouxel R, Grandjean B, et al. Activation of mirror-neuron system by erotic video clips predicts degree of induced erection: an fMRI study. Neuroimage 2008; 42(3): 1142-50.

[17] Moulier V, Mouras H, Pélégrini-Issac M, et al. Neuroanatomical correlates of penile erection evoked by photographic stimuli in human males. Neuroimage 2006; 33(2): 689-99.

[18] Kuban M, Barbarre HE, Blanchard R. A comparison of volume and circumference phallometry: response magnitude and method agreement. Arch Sex Behav 1999; 28: 345-59.

[19] Rosen RC. Genital blood flow measurement in the male: psychophysiological techniques. In: Rosen RC, Beck JG, Eds. Patterns of sexual arousal. NewYork, Guilford USA, 1988; 53-77.

[20] Redouté J, Stoléru S, Grégoire MC, et al. Brain processing of visual sexual stimuli in human males. Hum Brain Mapp 2000; 11(3): 162-77.
[21] Carmichael ST, Price JL. Connectional networks within the orbital and medial prefrontal cortex of macaque monkeys. J Comp Neurol 1996; 371(2): 179-07.

[22] Steers WD. Neural pathways and central sites involved in penile erection: neuroanatomy and clinical implications. Neurosci Biobehav Rev 2000; 24(5): 507-16.

[23] Critchley HD, Elliott R, Mathias CJ, Dolan RJ. Neural activity relating to generation and representation of galvanic skin conductance responses: a functional magnetic resonance imaging study. J Neurosci 2000; 20(8): 3033-40.

[24] Cechetto DF, Saper CB. Evidence for a viscerotopic sensory representation in the cortex and thalamus in the rat. J Comp Neurol 1987; 262(1): 27-45.

[25] Oppenheimer SM, Gelb A, Girvin JP, Hachinski VC. Cardiovascular effects of human insular cortex stimulation. Neurology 1992; 42(9): 1727-32.

[26] Georgiadis JR, Holstege G. Human brain activation during sexual stimulation of the penis. J Comp Neurol 2005; 493(1): 33-38.

[27] Penfield W, Rasmussen T. Sensorimotor representation of the body. The cerebral cortex of man. A clinical Study of Localization of Function. Macmillan, NewYork, USA; 1950.

[28] Kell CA, von Kriegstein K, Rösler A, Kleinschmidt A, Laufs H. The sensory cortical representation of the human penis: revisiting somatotopy in the male homunculus. J Neurosci 2005; 25(25): 5984-87.

[29] Bradley WE, Farrell DF, Ojemann GA. Human cerebrocortical potentials evoked by stimulation of the dorsal nerve of the penis. Somatosens Mot Res 1998; 15(2): 118-27.

[30] Carr L, Iacoboni M, Dubeau MC, Mazziotta JC, Lenzi GL. Neural mechanisms of empathy in humans: a relay from neural systems for imitation to limbic areas. Proc Natl Acad Sci U S A 2003; 100(9): 5497-502.

[31] Decety J, Perani D, Jeannerod M, et al. Mapping motor representations with positron emission tomography. Nature 1994; 371(6498): 600-2.

[32] Decety J, Grèzes J. The power of simulation: imagining one's own and other's behavior. Brain Res 2006; 1079(1): 4-14.

[33] Grèzes J, Decety J. Functional anatomy of execution, mental simulation, observation, and verb generation of actions: a metaanalysis. Hum Brain Mapp 2001 Jan; 12(1): 1-19.

[34] Stephan KM, Fink GR, Passingham RE, et al. Functional anatomy of the mental representation of upper extremity movements in healthy subjects. J Neurophysiol 1995; 73(1): 373-86.

[35] Buccino G, Binkofski F, Fink GR, et al. Action observation activates premotor and parietal areas in a somatotopic manner: an fMRI study. Eur J Neurosci 2001; 13(2): 400-04.

[36] Hanakawa T, Honda M, Okada T, Fukuyama H, Shibasaki H. Differential activity in the premotor cortex subdivisions in humans during mental calculation and verbal rehearsal tasks: a functional magnetic resonance imaging study. Neurosci Lett 2003; 347(3): 199-201.

[37] Porro CA, Francescato MP, Cettolo V, et al. Primary motor and sensory cortex activation during motor performance and motor imagery: a functional magnetic resonance imaging study. J Neurosci 1996; 16(23): 7688-98.

[38] Adolphs R, Damasio H, Tranel D, Cooper G, Damasio AR. A role for somatosensory cortices in the visual recognition of emotion as revealed by three-dimensional lesion mapping. J Neurosci 2000; 20(7): 2683-90.

[39] Ruby P, Decety J. Effect of subjective perspective taking during simulation of action: a PET investigation of agency. Nat Neurosci 2001; 4(5): 546-50.

[40] Avikainen S, Forss N, Hari R. Modulated activation of the human SI and SII cortices during observation of hand actions. Neuroimage 2002; 15(3): 640-6. 